\title{
Uma Abordagem Heurística para o Algoritmo PTS na Redução da PAPR em Sistemas OFDM
}

\section{A Heuristic Approach on PTS Algorithm for PAPR Reduction in OFDM Systems}

\author{
Caio Henrique Azolini Tavares ${ }^{1}$; Taufik Abrão ${ }^{2}$
}

\begin{abstract}
Resumo
Este trabalho analisa a redução da relação entre a potência de pico e potência média (PAPR) em sistemas com multiplexagem por divisão de frequência ortogonal (OFDM) sob a perspectiva da otimização heurística aplicada à topologia de transmissão por sequência parcial, visando a redução de sua complexidade computacional. Uma elevada PAPR em sinais OFDM acarreta em redução da eficiência energética dos amplificadores de alta potência (HPA) presentes no transmissor que, para evitar distorção no sinal amplificado, devem ter seu ponto de operação deslocado reduzindo a potência do sinal de entrada por um nível denominado input back-off (IBO). A técnica de transmissão por sequência parcial (PTS) para redução dos níveis de PAPR foi analisada e avaliada por meio de otimização heurística denominada enxame de partículas (PSO); os resultados foram comparados aos obtidos com o algoritmo PTS convencional (C-PTS) em termos da relação entre complexidade computacional e redução da PAPR obtida. Os resultados numéricos de simulação mostraram que ao utilizar o algoritmo PSOPTS é possível atingir resultados quase-ótimos em aproximadamente um quarto do tempo utilizado pelo C-PTS, tornando o primeiro mais adequado para utilização comercial, que usualmente envolvem sistemas robustos com um elevado número de subportadoras, o que necessariamente implica em uma alta PAPR.

Palavras-chave: Multiplexagem por Divisão de Frequência Ortogonal (OFDM); Relação entre Potência de Pico e Potência Média (PAPR); Sequência de Transmissão Parcial (PTS); Otimização Heurística; Otimização por Enxame de Partículas (PSO).
\end{abstract}

\begin{abstract}
This paper deals with peak-to-average power ratio (PAPR) reduction in orthogonal frequency division multiplexing (OFDM) systems under the perspective of heuristic optimization applied to the partial transmit sequence topology, seeking the reduction of computational complexity. We have analyzed and evaluated the partial transmit sequence (PTS) technique for reducing PAPR levels by means of heuristic optimization, namely particle swarm optimization (PSO). An elevated PAPR level on an OFDM signal implies an energy efficiency reduction of the high-power amplifiers (HPA) at the transmitter electronics, whose operating point must be moved in order to keep the output signal free from distortion; this is achieved by reducing the input signal power by a level known as input back-off (IBO). Numerical results have been compared with the conventional PTS (C-PTS) algorithm, especially regarding the trade-off between computational complexity versus PAPR reduction. Our numerical results have demonstrated that by using the PSO-PTS algorithm it is possible to achieve near optimal results in approximately a quarter of the time used by C-PTS, making the former more suitable for commercial applications that involve a large number of subcarriers, which implies in a high-PAPR OFDM signal.
\end{abstract}

Keywords: Orthogonal Frequency Division Multiplexing (OFDM); Peak-to-Average Power Ratio (PAPR); Partial Transmit Sequence (PTS); Heuristic optimization; Particle Swarm Optimization (PSO).

1 Graduado no curso de Engenharia Elétrica pela Universidade Estadual de Londrina; caio.tavares11@gmail.com

2 Docente do Departamento de Engenharia Elétrica da Universidade Estadual de Londrina - DEEL-UEL; taufik@uel.br 


\section{Introdução}

Os esquemas de transmissão com multiplexagem por divisão de frequência ortogonal (OFDM) estão presentes em muitas das tecnologias recentes de telecomunicações, tais como IEEE 802.11 (WiFi) (goldsmith, 2005), comunicações móveis de $4^{\text {a }}$ geração (Long Term Evolution - LTE) (gouda; hussien, 2013), IEEE 802.16 (WiMAX) e Digital Video Broadcasting - Terrestrial (DVB-T). Tais sistemas empregam modulação multiportadora através de algoritmos IFFT/FFT, com o objetivo de mitigar os efeitos dos canais com desvanecimento seletivo em frequência ao usar subportadoras de banda estreita, bem como incrementar a eficiência espectral. No entanto, uma das principais desvantagens do OFDM é sua alta relação entre a potência de pico e potência média (PAPR) devido à combinação das subportadoras moduladas (efeitos do módulo e fase) no domínio do tempo. Para lidar com essa alta PAPR nos transmissores, o ponto de operação do amplificador de alta-potência (HPA) deve ser reduzido para manter o sinal sem distorções, esta redução é chamada de região de back-off. Ao fazer isso, a eficiência do HPA é reduzida substancialmente, aumentando assim o consumo de energia, um efeito menos danoso do que a perda de linearidade durante a transmissão, que causa interferência inter-portadora (ICI) devido à perda de ortogonalidade, diminuindo o desempenho geral do sistema.

Por meio de simulações computacionais MonteCarlo realizadas ao longo deste trabalho, é possível predizer o comportamento da PAPR conforme o número de subportadoras e ordem de modulação aumentam. Além disso, como as tecnologias atuais em geral empregam um grande número de subportadoras para aumentar a eficiência espectral da transmissão, o problema da PAPR se torna uma séria barreira para atingir uma conexão eficiente em termos de alta taxa de dados e de eficiência energética.

Existem diversas formas de se obter a redução da PAPR em sinais OFDM, tais como clipping (sUDHA; BALAN; KUMAR, 2014), tone reservation (TR), tone injection (TI) (WATTANASUWAKULL; BENJAPOLAKUL, 2005),
(JIAO; LIU; WANG, 2008), (PHOOMCHUSAK; PIRAK, 2011), assim como a técnica de transmissão de sequência parcial (PTS) (INOUE; tSUTSUI; MIYANAGA, 2013), (PHETSOMphou; yoshizAWA; miyanaga, 2010). Dentre essas técnicas, a PTS atinge uma redução significativa da potência de pico do sinal OFDM sem introduzir distorção no sinal como a técnica de clipping. Não obstante, o esquema de PTS convencional requer um elevado poder computacional para se atingir bons resultados, o que pode torná-lo inviável em certos cenários de interesse prático e comercial. Uma alternativa para este problema é o emprego de heurística, tais como otimização por enxame de partículas (PSO) (HUNG; HUANG; YEH; TAN, 2008), otimização por colônia de formigas (ACO) (ABRÃo, 2013), (BAOCHENG; TIANE; ZENGHUI, 2012), algoritmo genético (GA) (LIANG; CHEN; HUANG; CHENG, 2009), entre outros, tendo em vista reduzir o tempo de execução do PTS convencional e simultaneamente atingir resultados quase-ótimos para a redução da PAPR.

Este artigo visa, portanto, obter uma caracterização da PAPR sob o método de otimização de transmissão por sequência parcial convencional, comparando-o com sua implementação heurística de otimização por enxame de partículas, levando em conta o tempo de execução e a redução de PAPR alcançada; deixando claro ao leitor o compromisso de tempo/desempenho do método analisado.

A organização do artigo foi feita da seguinte forma: Na Seção 2 são apresentados os modelos de sistema utilizados, tais como sinais OFDM e a definição de PAPR. A Seção 3 introduz a topologia de redução de PAPR através da transmissão por sequência parcial (PTS). Na Seção 4 é apresentado o algoritmo de otimização por enxame de partículas e seu uso no problema em questão. Os resultados numéricos são discutidos na Seção 5. Por fim, conclusões são apresentadas na Seção 6.

\section{Modelo do Sistema}

Um símbolo OFDM composto por $\mathrm{N}$ símbolos M-PSK pode ser descrito como: 


$$
\mathbf{x}=\frac{1}{\sqrt{N}} \sum_{i=0}^{N-1} s_{i} e^{\frac{j 2 \pi n i}{N}}, \quad 0 \leq n \leq N-1
$$

sendo $\mathrm{M}$ a ordem de modulação do símbolo PSK, $s_{i}$ o símbolo modulado em fase na i-ésima subportadora de $\mathrm{x}=[\mathrm{x} 1 \mathrm{x} 2 \ldots \mathrm{xi} \ldots \mathrm{xN}]$, N é o tamanho da FFT e $x \in \mathbf{C}{ }^{N x 1}$ é o vetor do símbolo OFDM.

A relação entre potência de pico e potência média de um símbolo OFDM é definida pela razão entre a potência máxima instantânea e a potência média tomada no período de um símbolo:

$$
\operatorname{PAPR}(\mathbf{x})=10 \log _{10}\left\{\frac{(\max [|\mathbf{x}[\mathrm{i}]|])^{2}}{\mathbb{E}\left[|\mathbf{x}[\mathrm{i}]|^{2}\right]}\right\}[\mathrm{dB}]
$$

Sendo $\mathbb{E}[$.$] o operador de esperança estatística,$ $\mathbf{x}$ [i] a i-ésima amostra do símbolo OFDM, sendo i de 1 até $\mathrm{N}$.

Para $\mathrm{N}$ ou $\mathrm{M}$ grande, a PAPR pode ser considerada um número aleatório, visto que existem $M^{N}$ possíveis símbolos com PAPR distintas. Portanto, a PAPR é melhor analisada pelo ponto de vista estatístico, através da função distribuição cumulativa complementar (CCDF).

$$
\begin{aligned}
\mathrm{CCDF} & =\operatorname{Pr}\left(\mathrm{PAPR}>\mathrm{PAPR}_{0}\right) \\
& =1-\operatorname{Pr}\left(\mathrm{PAPR} \leq \mathrm{PAPR}_{0}\right) \\
& =1-\left(1-e^{-P A P R_{0}}\right)^{N}
\end{aligned}
$$

sendo PAPR $_{0}$ o limiar de PAPR considerado.

\section{Transmissão por Sequência Parcial}

Na topologia de transmissão por sequência parcial convencional, o sinal modulado $s$ no transmissor é dividido em $\boldsymbol{M}$ ramos de comprimento N/M , sendo $\boldsymbol{M}$ o número de ramos. O vetor resultante é completado com zeros, a fim de retornar ao tamanho do sinal original, ou seja, $N$. Em seguida, cada ramo passa pela transformada inversa de Fourier (IFFT) e por fim é multiplicado por um fator de peso $p(i)$, denominado fator de rotação de fase, obtido de um conjunto de $W$ valores possíveis. O vetor que representa os $\boldsymbol{M}$ fatores de rotação de fase utilizados em determinada iteração é denominado vetor sequência de fase w. Após a multiplicação, os ramos são somados de forma a construir o símbolo OFDM completo; a PAPR resultante é avaliada e o algoritmo testa a próxima combinação de w. O algoritmo PTS convencional deve avaliar todas as $W^{\mathbf{M}}$ possíveis combinações de sequência de fase a fim de retornar o vetor de fase que minimiza a PAPR, problema que cresce exponencialmente com o número de ramos $\boldsymbol{M}$.

Ao rotacionar uma seção do sinal em uma determinada fase e posteriormente combinar todos os ramos, oalinhamento de fases do símbolo OFDM que causa a alta potência de pico pode ser mitigado e, também, a probabilidade de ocorrência de uma alta potência de pico é significativamente reduzida (HUNG; HUANG; YEH; TAN, 2008).

Além da elevada complexidade computacional, uma das desvantagens presentes ao utilizar esta técnica de redução de PAPR é a necessidade de se transmitir $\boldsymbol{M} \cdot \log _{\mathbf{2}} W$ bits de informação adicional referentes à sequência de rotação utilizada, impactando substancialmente a eficiência espectral do sistema. Entretanto, se trata de uma técnica de précodificação que não distorce o sinal de saída, apenas modifica sua combinação, além disso apresenta um bom espaço para otimização no processo de busca pelo melhor vetor de fase.

A Figura 1 representa uma implementação da técnica PTS utilizando 4 ramos em um sistema com 16 subportadoras. 
Figura 1- Representação do Funcionamento do Algoritmo PTS

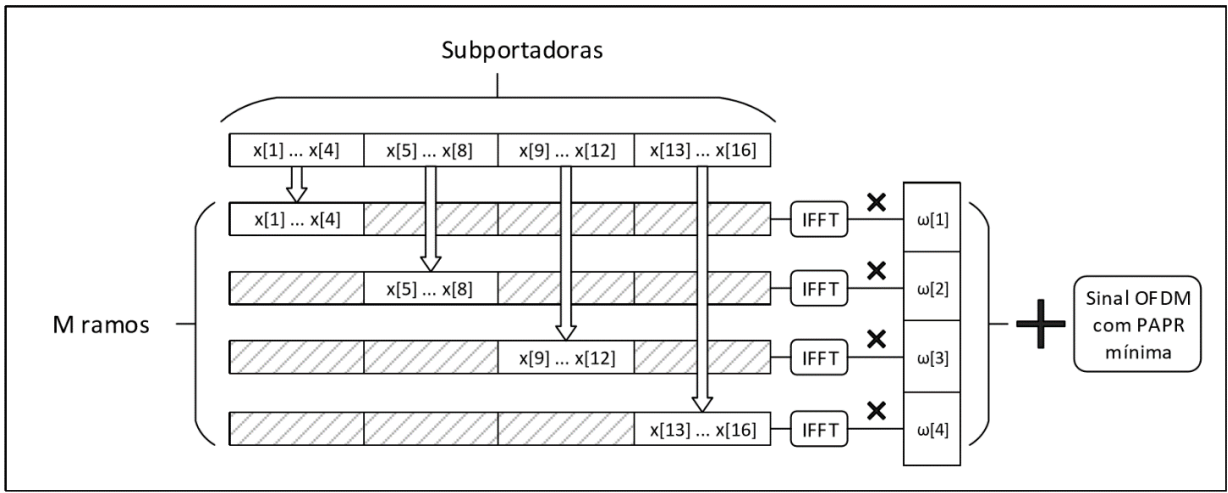

Fonte: Elaborada pelo autor

\section{Otimização Heurística}

A técnica de otimização por enxame de partículas (PSO) é bastante utilizada em diversos problemas distintos de otimização devido à sua simplicidade de implementação e configuração, quando comparada a outros métodos heurísticos como algoritmo genético (GA) que possuem mais parâmetros de entradas (BAI, 2010), (KATIYAR, 2010). Seu funcionamento se deve à observação do comportamento de grupos de pássaros durante o voo, cujas decisões a respeito da direção para onde prosseguir dependem de fatores de experiência individuais e sociais. De maneira simplificada, a técnica PSO gera uma população de "partículas" candidatas à solução do problema, com posições aleatórias ao longo do domínio da função, que para este problema em específico é discreto e, iterativamente, atualiza suas posições com base em seu rendimento, denominado fitness, o qual é calculado a partir da função objetivo que se quer otimizar, neste caso a minimização da PAPR de um dado símbolo OFDM. Cada partícula é representada por três indicadores individuais: sua posição espacial atual no domínio do problema, gerada aleatoriamente no início da primeira iteração; sua melhor posição obtida ao longo das iterações, denominada pbest e sua velocidade atual, utilizada na atualização de sua posição a cada nova iteração. Além disso, todas as partículas têm o conhecimento da melhor posição até a presente iteração obtida por qualquer membro da população, denominada gbest. Ao final de cada iteração, a velocidade de cada partícula é atualizada com base na seguinte equação:

$$
\begin{aligned}
v(t+1)= & I \cdot v(t)+c_{1} \cdot\left(p_{\text {best }}(t)-\theta(t)\right) \\
& +c_{2} \cdot\left(g_{\text {best }}(t)-\theta(t)\right)
\end{aligned}
$$

Onde $I$ representa o fator de inércia da partícula, que está ligado à sua velocidade anterior; $c_{1}$ representa o fator cognitivo, ou o peso da informação de sua melhor posição individual; $c$, representa o fator social, ou seja, o peso da informação da melhor posição global; $\theta(t)$ é a posição espacial da partícula na iteração $t$ e $v(t)$ é a velocidade da partícula na iteração $t$.

$\mathrm{O}$ algoritmo finaliza sua execução quando o melhor rendimento obtido ao longo das iterações atinge um limiar estabelecido previamente, aqui denominado threshold, ou quando o número máximo de iterações foi executado. No último caso, o algoritmo retorna a partícula associada à gbest como solução para o problema.

O funcionamento do PSO-PTS implementado pode ser melhor visualizado pelo procedimento descrito abaixo: 
1) Inicialização

a. Inicializar uma população de tamanho prédefinido com posições aleatórias e velocidade zero.

b. Avaliar a fitness de todas as partículas e salvar as melhores posições individuais (pbest) e a melhor global (gbest).

c. Atualizar a velocidade das partículas com base nos valores pbest e gbest, utilizando os valores definidos de inércia, $c_{1}$ e $c_{2}$.

2) Processo Iterativo

a. Atualizar a posição das partículas somando a posição anterior à velocidade nova calculada.

b. Avaliar a fitness das partículas. Comparar o rendimento obtido com o pbest de cada partícula e, caso necessário, atualizar pbest. Comparar melhor fitness obtido na iteração com gbest e, caso necessário, atualizar gbest.

c. Comparar gbest com o nível de threshold. Se gbest $\leq$ threshold retornar gbest e encerrar. Caso contrário, continuar ao passo 2-d.

d. Atualizar a velocidade das partículas com base nos valores pbest e gbest, utilizando os valores definidos de inércia, $c_{1}$ e $c_{2}$. Retornar ao passo 2-a.

3) Convergência

a. O algoritmo retorna a partícula gbest quando esta atinge o nível de threshold ou quando executa o número máximo de iterações permitido.

\section{Resultados Numéricos}

Para avaliar a eficácia do método PTS heurístico de redução da PAPR proposto, foram utilizadas simulações Monte-Carlo com a esperança de se obter uma tendência de comportamento para um grande número de símbolos OFDM, comparando o desempenho e tempo de execução do PTS convencional (C-PTS) e do PTS modificado (PSO-PTS).
No primeiro cenário, foram avaliados 10000 símbolos OFDM com 128 subportadoras e modulação QPSK. Considerando a redução média da PAPR ao longo da simulação, o algoritmo PSO-PTS atingiu $85,83 \%$ do desempenho obtido pelo C-PTS e, para isto, utilizou apenas cerca de $15 \%$ do tempo de execução. A curva complementar de distribuição cumulativa de probabilidade (CCDF) na Figura 2 mostra o desempenho obtido para o algoritmo PSOPTS, C-PTS e a distribuição de PAPR do símbolo OFDM original. O eixo y representa a probabilidade de um dado símbolo OFDM ter uma potência de pico $\mathrm{x}$ dB acima da potência média.

Figura 2 - Comparação de PAPR: PSO-PTS, C-PTS e OFDM original para modulação QPSK e 128 subportadoras

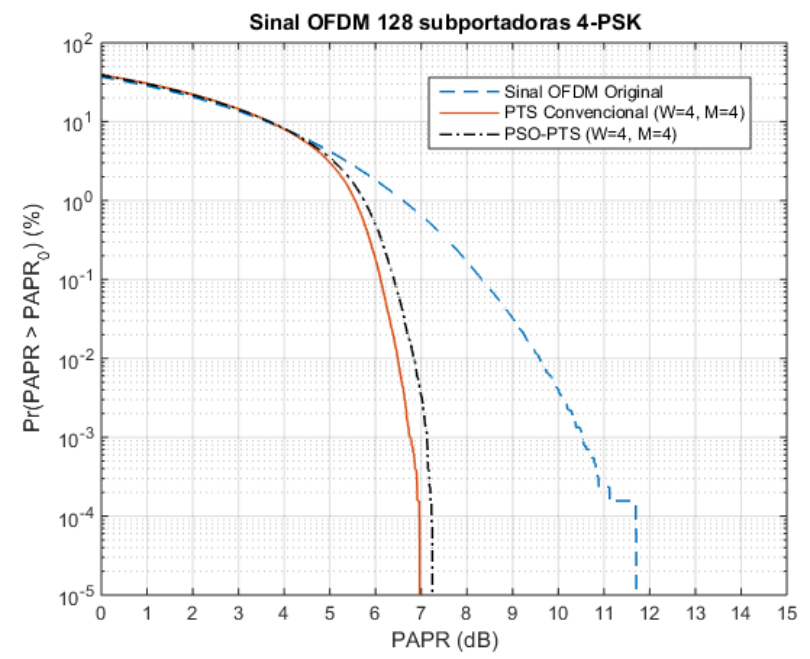

Fonte: Elaborada pelo autor

No segundo cenário foram simulados 10000 símbolos OFDM de 512 subportadoras e modulação 16-PSK. Novamente, considerando a PAPR média, por meio do algoritmo PSO-PTS foi possível obter 93,17\% do desempenho obtido com o algoritmo C-PTS, em cerca de $23 \%$ do tempo. Já quanto à PAPR máxima obtida ao longo de todos os símbolos simulados, o algoritmo PSO-PTS proposto obteve o mesmo desempenho do C-PTS, ambos convergindo em aproximadamente $8 \mathrm{~dB}$, cerca de $4 \mathrm{~dB}$ abaixo da PAPR do sinal OFDM original, conforme identificado na curva CCDF da Figura 3. 
Figura 3 - Comparação de PAPR: PSO-PTS, C-PTS e OFDM original para modulação 16-PSK e 512 subportadoras

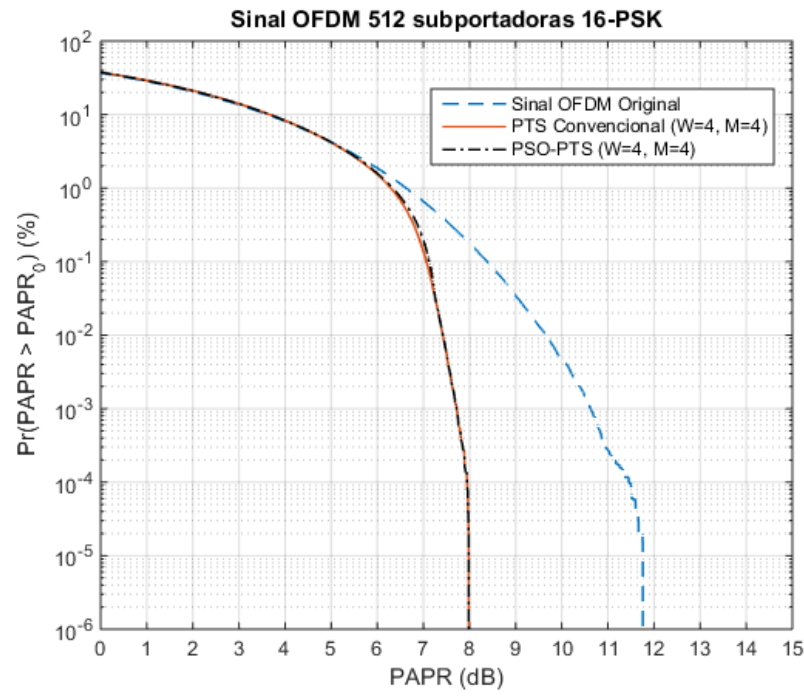

Fonte: Elaborada pelo autor

Considerando os dois cenários simulados, é possível afirmar que mesmo aumentando a complexidade do problema em termos de ordem de modulação e número de subportadoras, o algoritmo PSO-PTS manteve sua clara vantagem de tempo de execução, obtendo ainda resultados próximos do algoritmo convencional C-PTS.

\section{Conclusões}

Por meio da técnica de otimização por enxame de partículas (PSO), foi possível reduzir consideravelmente a complexidade computacional presente no método de transmissão por sequência parcial convencional (C-PTS) utilizado na redução de PAPR em sinais OFDM, obtendo ainda um desempenho quase-ótimo, ou seja, esta implementação heurística possui um trade-off vantajoso para o problema analisado.

Entretanto, deve ser observado que o algoritmo PSO requer que seus três parâmetros de entrada (inércia I, fator individual $c_{1}$ e fator social global $c_{2}$ ) sejam configurados criteriosa e cuidadosamente para cada classe/tipo de problema de otimização, a fim de se obter o máximo de seu desempenho no menor tempo de convergência possível. Desta forma, fica claro que uma metodologia sistemática de ajuste de parâmetros de entrada do algoritmo PSO tem essencial importância, em detrimento do ajuste puramente empírico. Além disso, ressalta-se que a comparação com outras técnicas heurísticas, tais como a otimização por colônia de formigas (ACO) ou algoritmo genético (GA), assim como outras topologias de redução de PAPR, em detrimento da técnica PTS, pode fornecer uma visão mais ampla a respeito do comportamento do problema em questão.

\section{Referências}

ABRÃO, T. (Ed.) Search algorithms for engineering optimization. Croatia: Intech, 2013.

BAI, Q. Analysis of particle swarm optimization algorithm. Computer and Information Science, Toranto, v. 3, n. 1, p.180-184, 2010.

BAOCHENG, W.; TIANE, W.; ZENGHUI, W. The implementation of parallel ant colony optimization algorithm based on MATLAB. In: GLOBAL CONGRESS ON INTELLIGENT SYSTEMS (GCIS), 3th., Wuhan, China. Proceeding... Wuhan, China, p.27-29, 2012.

GOLDSMITH, A. Wireless communications. Cambridge: Cambridge University Press, 2005.

GOUDA, M.; HUSSIEN, M. Partial transmit sequence PAPR reduction method for LTE OFDM systems. In: INTERNATIONAL CONFERENCE ON INTELLIGENT SYSTEMS MODELLING AND SIMULATIONS (ISMS), $4^{\text {th }} ., 2013$, Bangkok, Tailândia. Proceeding... Bangkok, Tailândia, p.507-512, 2013.

HUNG, H. L.; HUANG, Y. F.; YEH, C. M.; TAN, T. H. Performance of particle swarm optimization techniques on PAPR reduction for OFDM systems. In: IEEE INTERNATIONAL CONFERENCE ON SYSTEMS, MAN AND CYBERNETICS, 2008, Singapore. Proceeding... Singapore, 2014. p. 2390-2395. 
INOUE, Y.; TSUTSUI, H.; MIYANAGA, Y. Study of PAPR reduction using coded PTS in $8 \mathrm{x} 8$ MIMO-OFDM systems. In: INTERNATIONAL SYMPOSIUM ON INTELLIGENT SIGNAL PROCESSING AND COMMUNICATIONS SYSTEMS, 2013., Okinawa, Japan. Proceeding... Okinawa, Japan, p.363-368, 2013.

JIAO, Y. Z.; LIU, X. J.; WANG, X. A. A novel tone reservation scheme with fast convergence for PAPR reduction in OFDM systems. In: IEEE CONSUMER COMMUNICATIONS AND NETWORKING CONFERENCE, $5^{\text {th }}$., 2008, Las Vegas, Nevada. Proceeding ... Las Vegas, Nevada, p.398-402, 2008.

KATIYAR, S. A Comparative Study of Genetic Algorithm and the Particle Swarm Optimization. AKGEC International Journal of Technology, Delhi, v. 2, n. 2, p.21-24, 2010.

LIANG, H.; CHEN, Y. R.; HUANG, Y. F.; CHENG, C. H. A modified genetic algorithm PTS technique for PAPR reduction in OFDM systems. In: ASIA-PACIfiC CONFERENCE ON COMMUNICATIONS, $15^{\text {th }}$., 2009, Shanghai. Proceeding... Shanghai, p.182-185, 2009.

PHETSOMPHOU, D.; YOSHIZAWA, S.; MIYANAGA, Y. A partial transmit sequence technique for PAPR reduction in MIMO-OFDM systems. In: INTERNATIONAL SYMPOSIUM ON COMMUNICATIONS AND INFORMATION TECHNOLOGIES (ISCIT), 10 ${ }^{\text {th }}, 2010$, Tokio. Proceeding... Tokio, p.672-676, 2010.

PHOOMCHUSAK, P.; PIRAK, C. Adaptive tonereservation PAPR technique with optimal subcarriers allocation awareness for multi-user OFDMA systems. In: INTERNATIONAL CONFERENCE ON ADVANCED COMMUNICATION TECHNOLOGY (ICACT), 13 ${ }^{\text {th }}, 2011$, GangwonDo, Korea. Proceeding... Gangwon-Do, Korea. p.814-818, 2011.

SUDHA, V.; BALAN, S.; KUMAR, S. Performance analysis of PAPR reduction in OFDM system with distortion and distortion-less methods. In: INTERNATIONAL CONFERENCE ON COMPUTER COMMUNICATION AND INFORMATICS, 2014, Coimbatore, India. Proceeding... Coimbatore, India, p.1-4, 2014.
WATTANASUWAKULL, T.; BENJAPOLAKUL, W. PAPR reduction for OFDM transmission by using a method of tone reservation and tone injection. In: INTERNATIONAL CONFERENCE ON INFORMATION, COMMUNICATIONS AND SIGNAL PROCESSING, $5^{\text {th }}$, 2005, Bangkok, Thailand. Proceeding ... Bangkok, Thailand, p.273277, 2005.
Recebido em 20 Outubro, 2015 - Received on October 20, 2015 Aceito em 04 Abril, 2016-Accepted on April 04, 2016 
Tavares, C. H. A.; Abrão, T. 